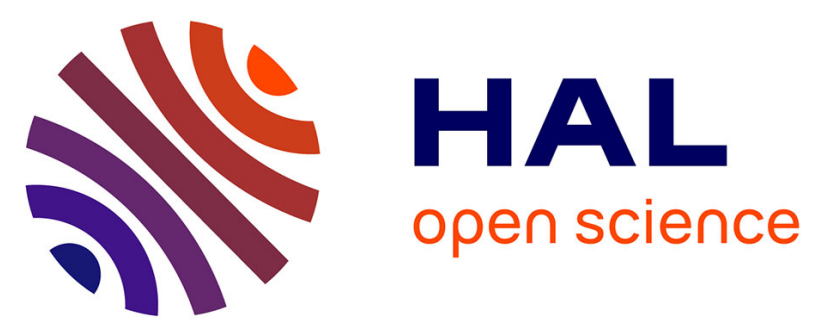

\title{
Energy-aware Adaptive Attitude Estimation Under External Acceleration for Pedestrian Navigation
}

\author{
Aida Makni, Hassen Fourati, Alain Kibangou
}

\section{To cite this version:}

Aida Makni, Hassen Fourati, Alain Kibangou. Energy-aware Adaptive Attitude Estimation Under External Acceleration for Pedestrian Navigation. IEEE/ASME Transactions on Mechatronics, 2016, 21 (3), pp.1366-1375. 10.1109/TMECH.2015.2509783 . hal-01241403

\section{HAL Id: hal-01241403 \\ https://inria.hal.science/hal-01241403}

Submitted on 24 Nov 2016

HAL is a multi-disciplinary open access archive for the deposit and dissemination of scientific research documents, whether they are published or not. The documents may come from teaching and research institutions in France or abroad, or from public or private research centers.
L'archive ouverte pluridisciplinaire HAL, est destinée au dépôt et à la diffusion de documents scientifiques de niveau recherche, publiés ou non, émanant des établissements d'enseignement et de recherche français ou étrangers, des laboratoires publics ou privés. 


\title{
Energy-aware Adaptive Attitude Estimation Under External Acceleration for Pedestrian Navigation
}

\author{
Aida Makni, Hassen Fourati and Alain Y. Kibangou
}

\begin{abstract}
In this paper, we consider the problem of rigid body attitude estimation under external acceleration using a small inertial/magnetic sensors module containing a triad of gyroscope, accelerometer, and magnetometer. The paper is focused on two main challenges. The first one concerns the attitude estimation during dynamic cases, in which external acceleration occurs. In order to compensate for such external acceleration, we design a quaternion-based adaptive Kalman filter q-AKF. Precisely, a smart detector is designed to decide whether the body is in static or dynamic case. Then, the covariance matrix of the external acceleration is estimated to tune the filter gain. The second challenge is related to the energy consumption issue of gyroscope. In order to ensure a longer battery life for the Inertial Measurement Units, we study the way to reduce the gyro measurements acquisition by switching on/off the sensor while maintaining an acceptable attitude estimation. The switching policy is based on the designed detector. The efficiency of the proposed scheme is evaluated by means of numerical simulations and experimental tests.
\end{abstract}

Index Terms-Attitude estimation, quaternion, adaptive Kalman filter, detection, dynamic and static cases, Energy management.

\section{INTRODUCTION}

Accurate tracking of rigid body attitude, i.e. the determination of the three-dimensional orientation from non-ideal strapdown sensors, is a requirement for ambulatory applications such as detection of unconstrained walking [1], pedestrian localization [2], [3], indoor navigation [4], [5], and human body trackers [6], [7]. Since several decades, it is usual to resort to Inertial Measurements Units (IMUs) composed of a triaxial gyroscope to measure angular velocity, a triaxial accelerometer to measure the sum of external acceleration and gravity, and a triaxial magnetic sensor to measure Earth's magnetic field [8]. Although these sensors can be used separately to infer the attitude, one rather tries to carry out an optimal fusion of their measurements in order to improve the estimation accuracy.

Using these sensors, several attitude estimation methods have been proposed in the literature. They are based on classical filtering methods such as Kalman filters (KFs) [9], [10], extended Kalman filters (EKFs) [11], or nonlinear observers [12], [13]. For instance, a quaternion Kalman filter (QKF) using an original linear observation model was introduced by Choukroun et al. [14]. Although these methods perform well enough during static cases, severe performance degradations are noticed during dynamic cases. Indeed, external accelerations occuring during dynamic cases are not taken into account when these methods are derived.

The authors are with Univ. Grenoble Alpes, CNRS, Gipsa-Lab, F-38000 and with Inria, Grenoble, France. (e-mails: aida.makni, hassen.fourati, alain.kibangou@gipsa-lab.fr).
To deal with this issue, some works considered explicitely the impact of external acceleration [15], [16], [17]. For instance, an adaptive EKF has been proposed in [15], where a diagonal matrix is added to the observation noise covariance matrix. However, it could not reflect accurately the influence of accelerations on the observation covariance matrix. Authors in [17] introduced a switching architecture to separate the two modes (low and high external acceleration). The effect of external acceleration is compensated for by setting the covariance matrix of accelerometer measurements to infinity. The approaches in [15], [16], [17] require the setting of thresholds according to the system dynamics which is very tricky in practice. The authors of [18] addressed the same problem by using an external acceleration model. The proposed algorithm behaves well for low to medium level external acceleration during short periods. Contrariwise, when high external acceleration occurs for long duration, this method is impaired by increasing errors. Definitely, efficient methods for attitude estimation, whatever the level and the duration of external acceleration, are still to be devised.

It is now well known that using gyroscope measurements seems to be crucial to overcome the limitation of accelerometers. However, gyroscopes are much more power consuming than accelerometers. As an example, the triaxial gyroscope L3GD20 consumes $6.1 \mathrm{~mA}[19]$ while the consumption of the triaxial accelerometer LIS3DH is around $11 \mu \mathrm{A}$ [20]. For battery-operated applications using IMUs in attitude estimation, such as Pedestrian Navigation Systems (PNS), Smartphones, and monitoring systems for elderly or visually impaired persons, the energy consumption of gyroscope is clearly a crucial issue. To the best to the authors knowledge, there is no work dealing with this issue in connection with the attitude estimation performance. Nowadays, a new generation of gyroscopes can be switched to operate in a low power mode (sleep mode) providing significant reduction of operating current (consumption around $1.5 \mathrm{~mA}$ ) [19]. It is therefore interesting to study how to decrease the use of gyroscope measurements by switching as often as possible to a sleep mode without a significant loss of performance during the attitude estimation process.

The main contributions of the paper can be stated as follows:

- A smart way for detecting dynamic case. A detection law is proposed to decide whether the body is in dynamic or static cases. As a consequence, the gyroscope can be switched to sleep mode (during static case) and a specific tuning of the process covariance matrix is proposed (see Fig. 1).

- A quaternion-based adaptive Kalman Filter (q-AKF) com- 
pensating the external acceleration effect. The main advantage of the proposed method is that the observation covariance matrix is adaptively tuned according to the filter residual when dynamic cases are detected (Fig. 1).

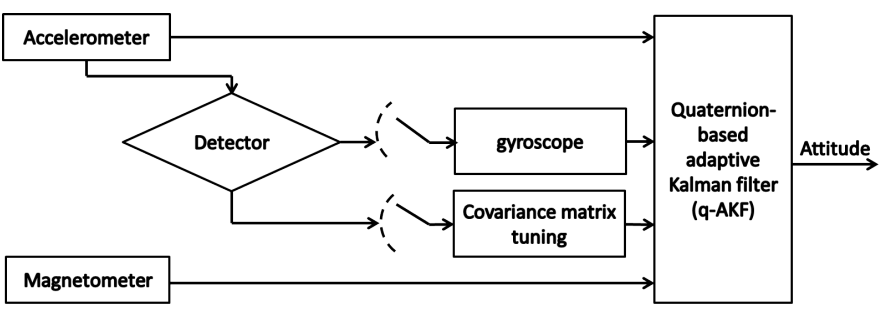

Fig. 1. Block diagram of the q-AKF employed for attitude estimation.

- A validation of the proposed approach in pedestrian navigation where the angular variations range is around $+/-180^{\circ}$ for yaw angle and $+/-90^{\circ}$ for roll and pitch angles, the angular velocity variations range is around $+/-600^{\circ} / \mathrm{s}$ and the acceleration norm range is around $+/-$ $8 g$. The expected estimation errors should be less than $10^{\circ}$ for the estimated Euler angles.

This paper is organized as follows: In Section II the problem of attitude estimation under external acceleration and energy consumption constraints is formulated. In Section III, the detection approach to separate static and dynamic cases is explained. In Section IV, the q-AKF is devised and then evaluated by means of numerical simulations (section $\mathrm{V}$ ) and experimental tests (section VI). Section VII provides some conclusions.

Notations: The skew matrix associated with a vector $x=$ $\left[\begin{array}{lll}x_{1} & x_{2} & x_{3}\end{array}\right]^{T} \in \mathbb{R}^{3}$ is defined as:

$$
\left[x^{\times}\right]=\left[\begin{array}{ccc}
0 & -x_{3} & x_{2} \\
x_{3} & 0 & -x_{1} \\
-x_{2} & x_{1} & 0
\end{array}\right] .
$$

A unit norm quaternion $q$ is defined by $q=\left[\begin{array}{ll}q_{0} & \vec{q}^{T}\end{array}\right]^{T}=$ $\left[\begin{array}{llll}q_{0} & q_{1} & q_{2} & q_{3}\end{array}\right]^{T} \in \mathbb{R}^{4}$. For two unit-norm quaternions $q_{a}=$ $\left[\begin{array}{cc}q_{a 0} & \vec{q}_{a}\end{array}\right]^{T}$ and $q_{b}=\left[\begin{array}{ll}q_{b 0} & \vec{q}_{b}\end{array}{ }^{T}\right.$, the quaternion product can be defined as:

$$
q_{a} \otimes q_{b}=\left[\begin{array}{cc}
q_{a 0} & -\vec{q}_{a}^{T} \\
\vec{q}_{a} & q_{a 0} I_{3}+\left[\vec{q}_{a} \times\right]
\end{array}\right]\left[\begin{array}{c}
q_{b 0} \\
\vec{q}_{b}
\end{array}\right],
$$

where $I_{3} \in \mathbb{R}^{3 \times 3}$ stands for the identity matrix. The complementary quaternion is defined as: $q^{-1}=\left[\begin{array}{llll}q_{0} & -q_{1} & -q_{2} & -q_{3}\end{array}\right]^{T}$ and we have the property : $q^{-1} \otimes q=\left[\begin{array}{llll}1 & 0 & 0 & 0\end{array}\right]^{T}$. More details about quaternion can be found in [21].

$C(q)$ stands for the rotation matrix from the Earth-fixed frame $N\left(X_{N}, Y_{N}, Z_{N}\right)^{1}$ (navigation frame) to the body-fixed frame $B\left(X_{B}, Y_{B}, Z_{B}\right)$. It is defined as:

$$
C(q)=\left[\begin{array}{ccc}
2 q_{0}^{2}+2 q_{1}^{2}-1 & 2 q_{1} q_{2}+2 q_{0} q_{3} & 2 q_{1} q_{3}-2 q_{0} q_{2} \\
2 q_{1} q_{2}-2 q_{0} q_{3} & 2 q_{0}^{2}+2 q_{2}^{2}-1 & 2 q_{2} q_{3}+2 q_{0} q_{1} \\
2 q_{1} q_{3}+2 q_{0} q_{2} & 2 q_{2} q_{3}-2 q_{0} q_{1} & 2 q_{0}^{2}+2 q_{3}^{2}-1
\end{array}\right]
$$

\footnotetext{
${ }^{1} X_{B}, Y_{B}$, and $Z_{B}$ axes point along each of the triad of sensors. The $X_{N^{-}}$ axis points to the true North. The $Z_{N}$-axis points towards the interior of the Earth, perpendicularly to the reference ellipsoid. The $Y_{N}$-axis completes the right-handed coordinate system, pointing East (NED: North, East, Down).
}

\section{PROBLEM FORMUlation}

Let us consider a rigid body with attitude represented by a quaternion $q$ and characterized by the kinematic equation [22]:

$$
\dot{q}=\frac{1}{2} q \otimes \bar{\omega},
$$

where $\bar{\omega}=\left[\begin{array}{ll}0 & \omega^{T}\end{array}\right]^{T}$ is the quaternion representation of angular velocity $\omega=\left[\begin{array}{lll}\omega_{x} & \omega_{y} & \omega_{z}\end{array}\right]^{T}$ expressed in $B$. Since there is no way to directly sense the attitude, it is usual to resort to data fusion approaches from a triad of sensors consisting of a triaxial gyroscope, a triaxial accelerometer, and a triaxial magnetometer [9], [11], [12], [13]. As commonly adopted in [23], [24], their outputs $y_{g}, y_{a}$, and $y_{m} \in \mathbb{R}^{3}$ are given by:

$$
\begin{aligned}
y_{g} & =\omega+\delta_{g}, \\
y_{a} & =C(q) G+a_{p}+\delta_{a}, \\
y_{m} & =C(q) m+\delta_{m},
\end{aligned}
$$

where $a_{p} \in \mathbb{R}^{3}$ denotes the external acceleration vector of the body (non-gravitational acceleration), $G=\left[\begin{array}{lll}0 & 0 & g\end{array}\right]^{T}$ is the gravity vector $\left(g=9.81 \mathrm{~m} / \mathrm{s}^{2}\right), \quad m=\left[\begin{array}{lll}m_{x} & m_{y} & m_{z}\end{array}\right]^{T}=$ $\left[\begin{array}{lll}\|m\| & \cos \theta \quad 0 \quad\|m\| \sin \theta\end{array}\right]^{T}$ represents the Earth's magnetic field vector measured in the Earth-fixed frame $N$ and $\theta$ is the inclinaison angle of the magnetic field. Actually, the theoretical model of the geomagnetic field $m$ may vary according to the location on Earth. However, $\|m\|$ and $\theta$ can be found by using the World Magnetic Model which takes the location on the earth into account [25]. Sensor noises $\delta_{g}, \delta_{a}$, and $\delta_{m}$ are assumed to be zero-mean white Gaussian noises mutually uncorrelated with the following covariance matrices $R_{g} I_{3}, R_{a} I_{3}$, and $R_{m} I_{3}$, respectively.

From Eq. (6), one can note that the accelerometer measurements are sensitive to the gravity vector $G$ and to $a_{p}$, the body external acceleration, that is usually unknown. Separating these two vectors is a tough task, in particular when the external acceleration increases. During high external acceleration, since the accelerometer output is affected by the motion, the attitude derived from such measurements is also affected, inducing a decrease of attitude estimation quality.

To overcome the limitations of accelerometer, the use of gyroscope seems to be crucial although it is much more power consuming than accelerometer. To reduce energy consumption, the authors proposed in [26] an arbitrary periodical switching between active and sleep modes of the sensor. In static case, ( ||$a_{p} \|$ is negligible compared to $\|G\|$ ), accelerometer and magnetometer are sufficient to achieve an accurate enough attitude estimation. Then, the gyroscope can be switched to sleep mode. In dynamic case (in presence of high external acceleration, i.e. non negligible values of $\left\|a_{p}\right\|$ compared to $\|G\|$ ), gyroscope should be used to compensate for the effects of the external acceleration. The main goal of this paper is to propose a smart way to detect dynamic cases and then to compensate for the effects of external acceleration in order to improve the attitude estimation while saving energy by reducing the use of gyroscope. 


\section{Detection OF MOTION}

The objective of this section is to define a detection law to decide whether, the body is in dynamic or static case, given a discrete sequence of accelerometer measurements $\left\{y_{a, k}, y_{a, k+1}, \ldots, y_{a, W+k+1}\right\}$. Mathematically, we can formalize the detection problem as a binary hypothesis testing problem, where the detector can choose between two hypotheses $H_{0}$ and $H_{1}$ defined as follows:

- $H_{0}$ : The body is in static case,

- $H_{1}$ : The body is in dynamic case.

In what follows, we give three ways to design the detector.

\section{A. Acceleration-based deterministic detector}

As stated in the previous section, during static cases, the external acceleration $\left\|a_{p, k}\right\|$ is negligible compared to $\|G\|$. Then, the norm of accelerometer measurements $\left\|y_{a, k}\right\|$ is almost equal to $\|G\|$. During dynamic cases, $\left\|y_{a, k}\right\|$ becomes much more higher or lower than $\|G\|$. Therefore, the detector can act by following the decision test below:

Test 1: Given $s_{1}$ and $s_{2}$ determined experimentally during a calibration step:

- if $s_{1} \leq\left\|y_{a, k}\right\| \leq s_{2} \Rightarrow H_{0}$ : static case,

- else $\Rightarrow H_{1}$ : dynamic case.

\section{B. Acceleration-based stochastic detector}

The previous detector acts instantaneously and can be prone to false detection due to outliers. In what follows, we design the detector such that the probability of detecting the dynamic events can be maximized given a certain false-alarm probability, i.e., given the probability to decide that the body is in dynamic case while it is not the case.

From Eqs. (3) and (6), and according to the detection hypotheses, we can note that the accelerometer output in discrete time, can be rewritten as:

- $y_{a, k}=g u_{k}+\delta_{a, k}$ under $H_{0}$,

- $y_{a, k}=g u_{k}+a_{p, k}+\delta_{a, k}$ under $H_{1}$.

where $u_{k} \in \mathbb{R}^{3}$ is the unit norm vector $\left(\| u_{k}||=1\right)$, given by:

$$
u_{k}=\left[\begin{array}{c}
2 q_{1, k} q_{3, k}-2 q_{0, k} q_{2, k} \\
2 q_{2, k} q_{3, k}+2 q_{0, k} q_{1, k} \\
2 q_{0, k}^{2}+2 q_{3, k}^{2}-1
\end{array}\right] .
$$

The idea is to define a detection function $f_{k}$, given $y_{a, k}$, and to compare it with a predefined threshold value $s$, such as:

- if $f_{k}\left(y_{a, k}\right)<s \Rightarrow H_{0}$ : static case,

- if $f_{k}\left(y_{a, k}\right) \geqslant s \Rightarrow H_{1}:$ dynamic case.

The hypothesis testing problem can be expressed by using the squared norm of $y_{a, k}$, which is defined as:

$$
\begin{aligned}
\left\|y_{a, k}\right\|^{2}= & g^{2}+\left\|\delta_{a, k}\right\|^{2}+2 g u_{k}^{T} \delta_{a, k} \quad \text { under } H_{0}, \\
\left\|y_{a, k}\right\|^{2}= & g^{2}+\left\|a_{p, k}\right\|^{2}+\left\|\delta_{a, k}\right\|^{2}+2 g u_{k}^{T} \delta_{a, k} \\
& +2 g u_{k}^{T} a_{p, k}+2 \delta_{a, k}^{T} a_{p, k} \quad \text { under } H_{1} .
\end{aligned}
$$

Note that, under $H_{0}$, since $\delta_{a, k}$ is assumed to be zero mean and independent of $u_{k}$, we get:

$$
E\left[\left\|y_{a, k}\right\|^{2}-g^{2}\right]=3 \sigma_{a}^{2}
$$

where $E[\bullet]$ denotes the expectation operation. In what follows, we approximate the mathematical expectation by:

$$
E\left[\left\|y_{a, k}\right\|^{2}-g^{2}\right]-3 \sigma_{a}^{2} \approx f_{k}=\frac{1}{W} \sum_{i=k-W+1}^{k}\left(\left\|y_{a, i}\right\|^{2}-g^{2}-3 \sigma_{a}^{2}\right) .
$$

We can note that (see Appendix. A):

$$
\begin{aligned}
E\left[f_{k}\right] & =0, \\
\sigma_{f}^{2}=\operatorname{var}\left[f_{k}\right] & =\frac{1}{W}\left(6 \sigma_{a}^{4}+4 g^{2} \sigma_{a}^{2}\right) .
\end{aligned}
$$

For a given scalar $n$, we know from the Chebyshev inequality that no more than $1 / n^{2}$ of the distribution values of the random variable $f_{k}$ can be more than $n$ standard deviations away from the mean, i.e. $P\left(\left|f_{k}\right| \geq n \sigma_{f}\right) \leq 1 / n^{2}$ [27]. In other hand, the probability of false-alarm is defined as:

$$
P F A=P\left(f_{k} \geq s / \text { the system is in static case }\right) .
$$

Therefore, by setting $n$ equal to $s / \sigma_{f}$, we can deduce that:

$$
P F A=\frac{1}{2} P\left(\left|f_{k}\right| \geq s\right) \leq \sigma_{f}^{2} / s^{2} .
$$

To ensure that the $P F A$ is lower than a pre-specified value $\alpha / 2$, we should select the threshold value as:

$$
s=\sqrt{\frac{\sigma_{f}^{2}}{\alpha}} .
$$

Test 2: Given a probability of the false-alarm $\alpha$ :

- if $f_{k}\left(y_{a, k}\right)<\sqrt{\frac{\sigma_{f}^{2}}{\alpha}} \Rightarrow H_{0}$ : static case,

- if $f_{k}\left(y_{a, k}\right) \geqslant \sqrt{\frac{\sigma_{f}^{2}}{\alpha}} \Rightarrow H_{1}:$ dynamic case.

\section{Acceleration-based hybrid detector}

The detection function $f_{k}$ can be too smooth and then induce a latency on the detection of some events. Indeed, in the construction of the function $f_{k}$ all measurements have the same weights. In order to give more weight to the current value, we suggest to combine the two detectors previously proposed:

Test 3: Given $s_{1}, s_{2}$, and $\alpha$ :

- if $f_{k}\left(y_{a, k}\right)<\sqrt{\frac{\sigma_{f}^{2}}{\alpha}}$ and $s_{1} \leq\left\|y_{a, k}\right\| \leq s_{2} \Rightarrow H_{0}$ : static case,

- else $\Rightarrow H_{1}$ : dynamic case.

\section{Adaptive Kalman Filter for ATtitude ESTIMATION}

In this section we propose a filtering approach for rigid body attitude estimation in two cases :

- standard energy consumption mode: the gyroscope is used at each instant (it is on active mode during the observation window);

- low energy consumption mode: the gyrospcope is used only if needed. In this case, the gyroscope switches between the active and sleep modes.

The necessary process and observation models for the filter design are defined in the following. 


\section{A. The process model}

In discrete-time, the differential equation describing the kinematics of the attitude in terms of quaternion (4), can be written as [14]:

$$
q_{k+1}=\Phi_{k} q_{k}+w_{k},
$$

where $q_{k}$ is the quaternion representing the attitude, $\Phi_{k}$ is the matrix containing the angular velocity vector $y_{g, k}$ :

$$
\begin{gathered}
\Phi_{k}=\exp \left(\Omega_{k} \Delta t\right), \\
\Omega_{k}=\frac{1}{2}\left[\begin{array}{cc}
0 & -y_{g, k}^{T} \\
y_{g, k} & -\left[y_{g, k}^{\times}\right.
\end{array}\right],
\end{gathered}
$$

where $\Delta t$ is the sampling period and $w_{k} \sim \mathscr{N}\left(0, Q_{k}\right)$ is the process noise defined as:

$$
w_{k}=-\frac{\Delta t}{2} \Xi\left(q_{k}\right) \delta_{g, k}
$$

with

$$
\Xi\left(q_{k}\right)=\left[\begin{array}{c}
-\vec{q}_{k}^{T} \\
{\left[\vec{q}_{k}{ }^{\top}\right]+q_{0, k} I_{3 \times 3}}
\end{array}\right] .
$$

We can note that the process model is mainly fed by gyroscope measurements. However, during static cases, it is reasonable to adopt a simple random walk model driven by a white Gaussian noise $w_{k}^{\prime}$ with a covariance matrix $Q_{k}^{\prime}$. In such case, we get:

$$
q_{k+1}=q_{k}+w_{k}^{\prime} .
$$

Let define the binary variables $\beta$ and $\gamma_{k}$ as:

- $\beta=0$ when the standard energy consumption mode is activated;

- $\beta=1$ when the low energy consumption mode is activated;

- $\gamma_{k}=0$ when the rigid body is in static case at time $k$;

- $\gamma_{k}=1$ when the rigid body is in dynamic case at time $k$. The models (18) and (23) give rise to the generic model:

$$
q_{k+1}=A\left(\gamma_{k}, \beta\right) q_{k}+b\left(\gamma_{k}, \beta\right),
$$

where $A\left(\gamma_{k}, \beta\right)$ and $b\left(\gamma_{k}, \beta\right)$ represent respectively the transition matrix and the process noise vector, defined as:

$$
\begin{aligned}
& A\left(\gamma_{k}, \beta\right)=\left(1-\beta\left(1-\gamma_{k}\right)\right) \Phi_{k}+\beta\left(1-\gamma_{k}\right) I_{4}, \\
& b\left(\gamma_{k}, \beta\right)=\left(1-\beta\left(1-\gamma_{k}\right)\right) w_{k}+\beta\left(1-\gamma_{k}\right) w_{k}^{\prime} .
\end{aligned}
$$

The covariance matrix of the process noise $b\left(\gamma_{k}, \beta\right)$ is defined as:

$$
\bar{Q}_{k}\left(\gamma_{k}, \beta, q_{k}\right)=\left(1-\beta\left(1-\gamma_{k}\right)\right) Q_{k}+\beta\left(1-\gamma_{k}\right) Q_{k}^{\prime} .
$$

We can note that in the low energy consumption mode $(\beta=1)$, the process model switches according to the static or dynamic case of the rigid body. Since gyroscope measurements seem to be mandatory only during dynamic cases, we can save energy by switching-off the gyroscope during static cases. The estimation process is then only fed by the accelerometer and the magnetometer [28], [29]. The switching process is governed by the decision test derived in the previous section.

\section{B. Observation model}

Let us consider the quaternion vectors $Y_{a, k}=\left[\begin{array}{ll}0 & y_{a, k}^{T}\end{array}\right]^{T}, \bar{G}=$ $\left[\begin{array}{ll}0 & G^{T}\end{array}\right]^{T}, Y_{m, k}=\left[\begin{array}{ll}0 & y_{m, k}^{T}\end{array}\right]^{T}$ and $\bar{m}=\left[\begin{array}{ll}0 & m^{T}\end{array}\right]^{T}$ related to $y_{a, k}$, $G, y_{m, k}$, and $m$, respectively.

$Y_{a, k}$ and $Y_{m, k}$ depend on the quaternion $q_{k}$ as follows [30]:

$$
\begin{aligned}
& Y_{a, k}=q_{k}^{-1} \otimes \bar{G} \otimes q_{k}, \\
& Y_{m, k}=q_{k}^{-1} \otimes \bar{m} \otimes q_{k} .
\end{aligned}
$$

Let left multiply both sides of Eqs. (28) and (29) by $q_{k}$. Then, the difference between the two sides in each obtained equation leads to the following quaternion pseudo-observation model [14], [31]:

$$
0_{8 \times 1}=H_{k} q_{k}+v_{k},
$$

where

$$
H_{k}=\left(\begin{array}{c}
H_{1, k} \\
H_{2, k}
\end{array}\right)
$$

with

$$
\begin{aligned}
H_{1, k} & =\frac{1}{2}\left(\begin{array}{cc}
0 & -\left(y_{a, k}-G\right)^{T} \\
\left(y_{a, k}-G\right) & -\left[\left(y_{a, k}+G\right)^{\times}\right]
\end{array}\right), \\
H_{2, k} & =\frac{1}{2}\left(\begin{array}{cc}
0 & -\left(y_{m, k}-m\right)^{T} \\
\left(y_{m, k}-m\right) & -\left[\left(y_{m, k}+m\right)^{\times}\right]
\end{array}\right),
\end{aligned}
$$

and

$$
v_{k}=\left(\begin{array}{c}
w_{a c c, k}^{q} \\
w_{m g, k}^{q}
\end{array}\right)=-\frac{1}{2} \Xi\left(q_{k}\right)\left(\begin{array}{c}
a_{p, k}+\delta_{a, k} \\
\delta_{m, k}
\end{array}\right) .
$$

One can note that the statistical characteristics of the quaternion-dependent noise, related to the magnetometer $w_{m g, k}^{q} \sim \mathscr{N}\left(0, R_{m g, k}\right)$, are known. However, those of the noise $w_{a c c, k}^{q} \sim \mathscr{N}\left(r_{k}, R_{a c c, k}\right)$, related to the accelerometer are unknown when external acceleration $a_{p, k}$ is contained in the accelerometer measurements $y_{a, k}$. Therefore, $r_{k}$ and $R_{a c c, k}$ will be adaptively estimated in the filter. Then, in the following, we considered $\hat{R}_{a c c, k}$ and $\hat{r}_{k}$ instead of $R_{a c c, k}$ and $r_{k}$.

\section{Online estimation of $\hat{R}_{a c c, k}$}

The external acceleration $a_{p, k}$ is unknown in the observation noise (34). Since the sensor noise $\delta_{a, k}$ is assumed to be a zeromean white Gaussian noise, the expectation value of $w_{a c c, k}^{q}$ in (34), is given by:

$$
E\left[w_{a c c, k}^{q}\right]=-\frac{1}{2} \Xi\left(q_{k}\right) a_{p, k} .
$$

Assuming that $\Xi\left(q_{k}\right)$ is full column rank, an estimate of the external acceleration can be obtained as:

$$
\hat{a}_{p, k}=-2 \Xi^{\dagger}\left(q_{k}\right) E\left[w_{a c c, k}^{q}\right] .
$$

Knowing that $0_{4 \times 1}=H_{1, k} q_{k}+w_{a c c, k}^{q}$, under ergodicity assumption we can approximate the expectation $E\left[w_{a c c, k}^{q}\right]$ by the arithmetical mean $\frac{1}{N} \sum_{j=k-N+1}^{k} r_{j}$, with $r_{j}=-H_{1, j} q_{j}$. Since the actual state is unknown we substitute it by its a priori estimate $\hat{q}_{k / k-1}$ giving rise to the residuals $r_{j}=-H_{1, j} \hat{q}_{j / j-1}$. Therefore:

$$
\hat{a}_{p, k}=-2 \Xi^{\dagger}\left(\hat{q}_{k / k-1}\right) \hat{r}_{k} .
$$


with

$$
\hat{r}_{k}=\frac{1}{N} \sum_{j=k-N+1}^{k} r_{j}=-\frac{1}{N} \sum_{j=k-N+1}^{k} H_{1, j} \hat{q}_{j / j-1} .
$$

The covariance matrix of external acceleration is then approximated by:

$$
\hat{R}_{p, k}=\left\|\hat{a}_{p, k}\right\|^{2} I_{3}
$$

We can then deduce that:

$$
\hat{R}_{a c c, k}=\frac{1}{4} \Xi\left(\hat{q}_{k / k-1}\right)\left(R_{a}+\hat{R}_{p, k}\right) \Xi\left(\hat{q}_{k / k-1}\right)^{T} .
$$

In the case of standard consumption mode $(\beta=0)$, we can assume that during static case the process model is robust enough to restrict the adaptation of the covariance matrix to dynamic case, i.e.:

$$
\hat{R}_{a c c, k}=\frac{1}{4} \Xi\left(\hat{q}_{k / k-1}\right)\left(R_{a}+\gamma_{k} \hat{R}_{p, k}\right) \Xi\left(\hat{q}_{k / k-1}\right)^{T} .
$$

Moreover, from (40) and (41), $\hat{R}_{a c c, k}$ can be expressed as:

$$
\hat{R}_{a c c, k}=\frac{1}{4} \Xi\left(\hat{q}_{k / k-1}\right)\left[R_{a}+\left(\gamma_{k}+\beta\left(1-\gamma_{k}\right)\right) \hat{R}_{p, k}\right] \Xi\left(\hat{q}_{k / k-1}\right)^{T} .
$$

\section{Filter design}

Based on the process and the observation models derived in the previous subsections, the following quaternion-based adaptive Kalman filter (q-AKF) is devised. We assume here that every unknown variable in the model can be replaced by its best available estimate. Then, the steps of the proposed q-AKF can be summarized as follows:

1) Initialize the state estimate $\hat{q}_{0 / 0}$, the error covariance matrix $P_{0 / 0}, \gamma_{0}$, and choose $\beta$.

For $k \geq 1$ :

2) Compute the a priori state estimate

$$
\hat{q}_{k / k-1}=A\left(\gamma_{k}, \beta\right) \hat{q}_{k-1 / k-1} \text {. }
$$

3) Compute the a priori error covariance estimate

$$
P_{k / k-1}=A\left(\gamma_{k}, \beta\right) P_{k-1 / k-1} A\left(\gamma_{k}, \beta\right)^{T}+\bar{Q}_{k}\left(\gamma_{k}, \beta, q_{k}\right),
$$

where $\bar{Q}_{k}\left(\gamma_{k}, \beta, q_{k}\right)$ is defined in (27) and in which $Q_{k}$ is computed such as [14]:

$$
Q_{k}=\frac{\Delta t^{2}}{4} \Xi\left(\hat{q}_{k-1 / k-1}\right) R_{g} \Xi\left(\hat{q}_{k-1 / k-1}\right)^{T},
$$

where $\Delta t$ stands for the sampling period and $Q_{k}^{\prime}$ is fixed a priori.

4) Compute the Kalman gain

$$
K_{k}=P_{k / k-1} H_{k}\left(H_{k} P_{k / k-1} H_{k}^{T}+R_{k}\right)^{-1},
$$

where

$$
R_{k}=\left(\begin{array}{c}
\hat{R}_{a c c, k} \\
R_{m g, k}
\end{array}\right),
$$

where $\hat{R}_{a c c, k}$ is given by (42) and

$$
R_{m g, k}=\frac{1}{4} \Xi\left(\hat{q}_{k / k-1}\right) R_{m} \Xi\left(\hat{q}_{k / k-1}\right)^{T} .
$$

$H_{k}$ is defined in (31) and $R_{m}$ is the covariance matrice of magnetometer noise.

5) Compute the a posteriori state estimate:

$$
\hat{q}_{k / k}=\hat{q}_{k / k-1}-K_{k}\left(H_{k} \hat{q}_{k / k-1}+\left(\begin{array}{c}
\hat{r}_{k} \\
0
\end{array}\right)\right) .
$$

6) Compute the a posteriori error covariance estimate

$$
P_{k / k}=\left(I-K_{k} H_{k}\right) P_{k / k-1} .
$$

\section{Simulation Results}

This section aims to illustrate the performance of the designed q-AKF. Some numerical simulations were carried out under MATLAB to estimate a rigid body attitude.

\section{A. Simulation setup}

In the first step, the following angular rate values issued from the gyroscope are simulated:

$\omega_{k}=\left[\begin{array}{lll}2 \cos (1,5 k) & -2 \sin (0.9 k) & 1.5 \cos (1.2 k)\end{array}\right]^{T}$. Then, the discrete-time kinematic equation (18) and the angular velocity $\omega_{k}$ were used to generate the sequence of quaternion $q_{k}$ over $10 \mathrm{~s}$, which will be considered later as a reference to compare it with the estimated quaternion from the qAKF. In the second step, the accelerometer and magnetometer outputs were generated using (6), (7), respectively. The rotation matrix in (3) computed using the quaternion $q_{k}$. The sampling rate was chosen as $100 \mathrm{~Hz}$ for all measurements. To represent the sensor imperfections, a random zero-mean white Gaussian noise was added for each sensor measurements, with a standard deviation given in Table I. A sequence of external acceleration $a_{p, k}$ (see the top of Fig. 2(a)) is added to the accelerometer output according to (6). Two different

TABLE I

CHARACTERISTICS OF THE VARIOUS NOISES FOR SENSOR MEASUREMENTS

\begin{tabular}{llll}
\hline \hline Sensors & Parameters & $\begin{array}{l}\text { Standard } \\
\text { deviations }\end{array}$ & Units \\
\hline Accelerometer & $\delta_{a}$ & 0.01 & $\mathrm{~m} / \mathrm{s}^{2}$ \\
Magnetometer & $\delta_{m}$ & 0.05 & $\mathrm{Gauss}$ \\
Gyroscope & $\delta_{g}$ & 0.05 & $\mathrm{rad} / \mathrm{s}$ \\
\hline
\end{tabular}

quaternions are considered to initialize the reference model $\left(q(0)=\left[\begin{array}{llll}0.3 & -0.6 & 0.75 & 0.1\end{array}\right]^{T}\right)$ as well as the $\mathrm{q}-\mathrm{AKF}$ $\left(\hat{q}(0)=\left[\begin{array}{llll}1 & 0 & 0 & 0\end{array}\right]^{T}\right)$ in this simulation. Notice that this choice allows us to illustrate the convergence of the $\mathrm{q}-\mathrm{AKF}$ even though it was initialized far enough from the reference states. The initial estimation error covariance matrix was chosen such as:

$$
P_{0 / 0}=0.1 I_{4 \times 4} .
$$

We evaluate the performance of the proposed method by computing Euler angles (roll, pitch, yaw) ${ }^{2}$. Then the quality of attitude estimation is evaluated according to the Root Mean

\footnotetext{
${ }^{2}$ The definitions of Euler angles are assumed to be the same as those of the airplane/ship convention estimation errors. Recall that Euler angles are obtained using the mathematical transformation of estimated quaternion [32]
} 
Square Error (RMSE) values of Euler angles. Thus, the RMSE is given by:

$$
\text { RMSE }=\sqrt{\frac{1}{T} \sum_{t=0}^{T} x_{\text {angle }}^{2}(t),}
$$

where $T$ stands for the time interval and $x_{\text {angle }}$, angle $\in\{$ pitch,roll,yaw $\}$ is the computed error between the estimated angle and the reference one, which can be expressed by:

$$
x_{\text {roll }}=\phi-\hat{\phi}, \quad x_{\text {pitch }}=\theta-\hat{\theta} \quad \text { or } \quad x_{\text {yaw }}=\psi-\hat{\psi},
$$

where $\phi, \theta, \psi$ are respectively the reference roll, pitch and yaw angles and $\hat{\phi}, \hat{\theta}$ and $\hat{\psi}$ are the estimated angles. MonteCarlo simulations of 100 independent runs were realized in all simulation cases. For online estimation of $\hat{R}_{a c c, k}$, as described in (42), $N$ was set equal to 100 .

\section{B. Filter performance in standard energy consumption mode $(\beta=0)$}

To show the improvements of the $\mathrm{q}-\mathrm{AKF}$, this latter is compared to the QKF (Quaternion Kalman Filter) developed in [14]. Both designed filters have a standard linear Kalman filter structure with a state dependent covariance matrix of process noise. The difference lies in the adaptive algorithm and the detection law proposed in this work. Fig. 2 depicts the evolution of absolute value of Euler angles estimation errors as in Eq. (53). For more clarity, two different scales are used, one for the full simulation period (see Fig. 2(a)) and a second for the first $50 \mathrm{~ms}$ in order to illustrate the convergence behavior (see Fig. 2(b)). Our aim is to illustrate the high convergence behavior in the time. We can see that both algorithms converge similarly but the q-AKF provides the best performance during the simulation period. The RMSE values of Euler angles is

TABLE II

RMSE OF EULER ANGLES ESTIMATION (Q-AKF VS QKF)

\begin{tabular}{cccc}
\hline \hline & Roll $\left(^{\circ}\right)$ & Pitch $\left(^{\circ}\right)$ & Yaw $\left(^{\circ}\right)$ \\
\hline q-AKF & 0.3346 & 0.5524 & 0.8562 \\
\hline QKF & 2.3174 & 2.5649 & 1.7616 \\
\hline
\end{tabular}

even two to seven times better than with QKF (see Table II). The QKF losses performance during dynamic cases. This result was expected since the QKF doesn't take into account the effect of external acceleration in its design. The q-AKF is more efficient since the external acceleration is taken into account due to the adaptive algorithm part. The detector of the motion seems to be also efficient enough. For this purpose, Fig. 3 (top) depicts the evolution of the detection function $f_{k}$ used in Test 2 and 3 (here we restrict the study to Test 2) with the detection threshold $s$. A zoomed part of this figure is also shown in the middle. We can see that the evolution of $f_{k}$ follows the chosen profile of external acceleration depicted in the top of Fig. 2(a). According to this function, the detected mode is depicted in Fig. 3 (bottom) where the considered binary function is equal to 1 during dynamic cases and zero else. To show the detector performance, we plotted the Detection Probability $P D$ versus the threshold value $\alpha$ of the

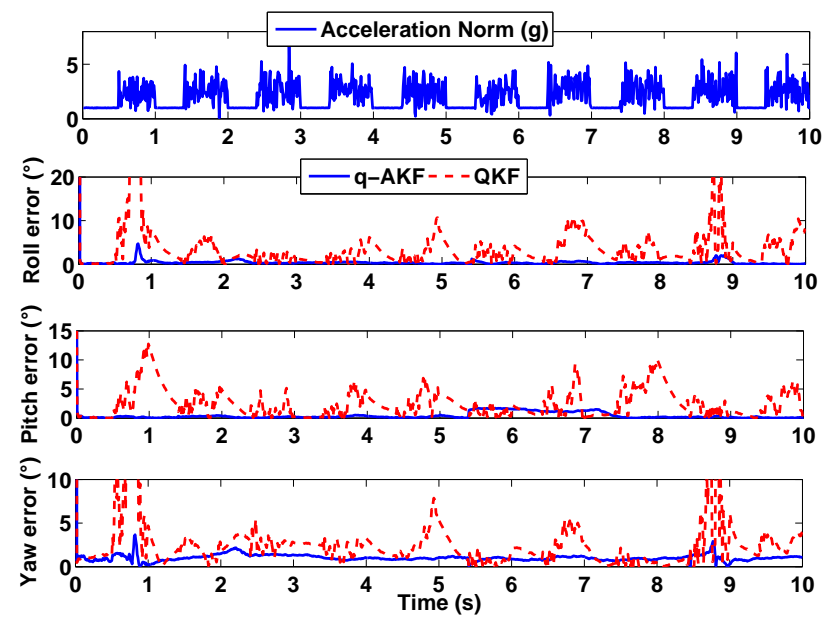

(a)
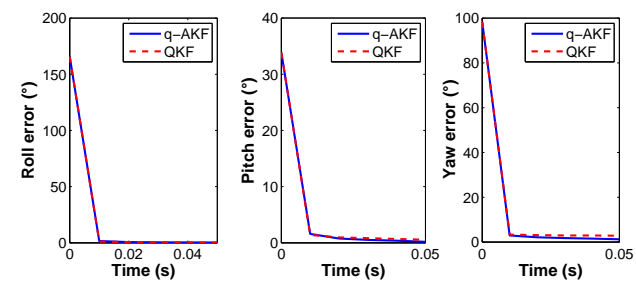

(b)

Fig. 2. Euler angles estimation errors: (a) full simulation period (b) zoomed period (between 0 and $0.05 \mathrm{~s}$ )
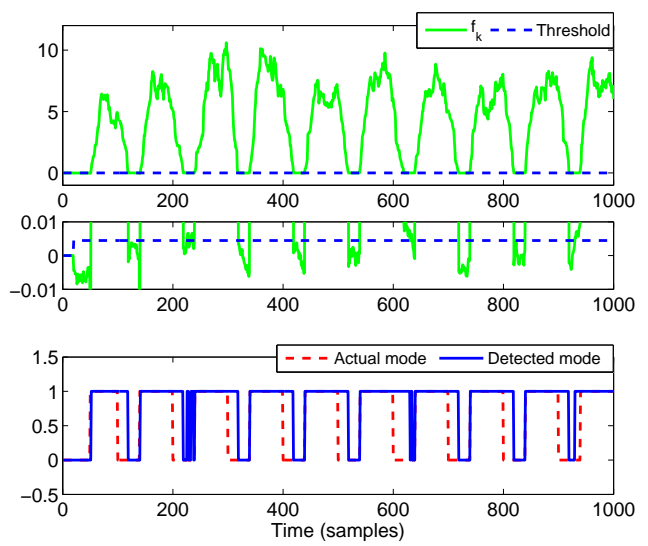

Fig. 3. External acceleration detection

false-alarm Probability $P F A$ for various values of the sliding window $W$. It can be seen in Fig. 4 that the $P D$ is monotonic with increasing $\alpha$ and it is high even if the PFA increases. We conclude that the developed detector is robust enough and presents a good performance. Moreover, it is clear that the detection law is more efficient when the size of the window $W$ increases but from 20 the improvement is no longer significant. In other side, to study the q-AKF performance under various dynamic cases, a simulation test different from the first one in terms of the magnitude and duration of external accelerations was performed. Fig. 5 shows Euler angles estimation errors of the $\mathrm{q}-\mathrm{AKF}$ and QKF. We note that the estimation errors with the q-AKF are kept low even when external accelerations take 


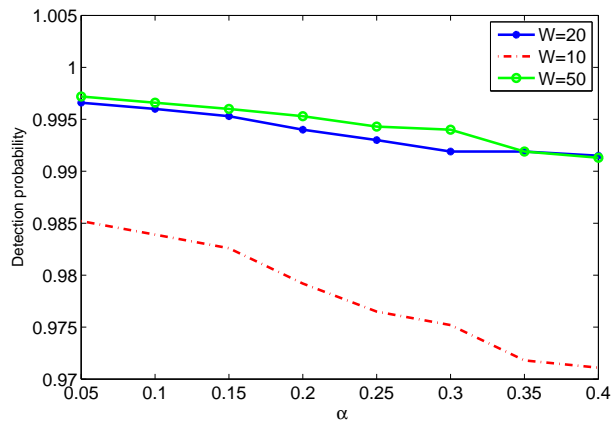

Fig. 4. Detection performance via detection probability

place for a long duration (between 30 and $50 \mathrm{~s}$ for example) and with a constant magnitude $(5 \mathrm{~g})$.

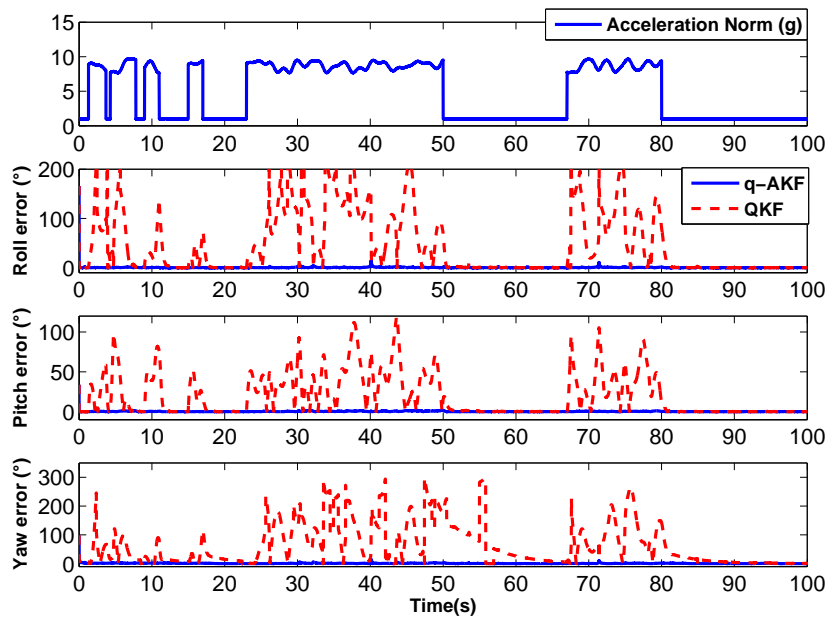

Fig. 5. Euler angles estimation errors for QKF and q-AKF under long external accelerations duration

C. Filter performance in low energy consumption mode ( $\beta=$ 1)

In this subsection, we consider the case of low energy consumption mode by reducing the use of gyroscope in attitude estimation (see Section III and IV). The switches between the gyroscope functioning modes are driven by the decision tests derived in the previous sections. The parameters $W$ and $\alpha$, used for computing the detection function $f_{k}$ and the threshold value $s$, are respectively chosen equal to 20 and 0.09 in Tests 2 and 3 while $s_{1}=0.996$ and $s_{2}=1.004$ in Tests 1 and 3. Fig. 6 and Table III show the estimation errors of Euler angles and their RMSE values in each test case (Test 1, 2 and 3 proposed in Section III). It is clear, from Fig. 6 and the RMSE values, that using the $\mathrm{q}-\mathrm{AKF}$ with Test 3 provides the best attitude determination. This Test allows the q-AKF to be more robust to external acceleration. Moreover, the nondetections inherent to Test 1 and Test 2 are compensated.

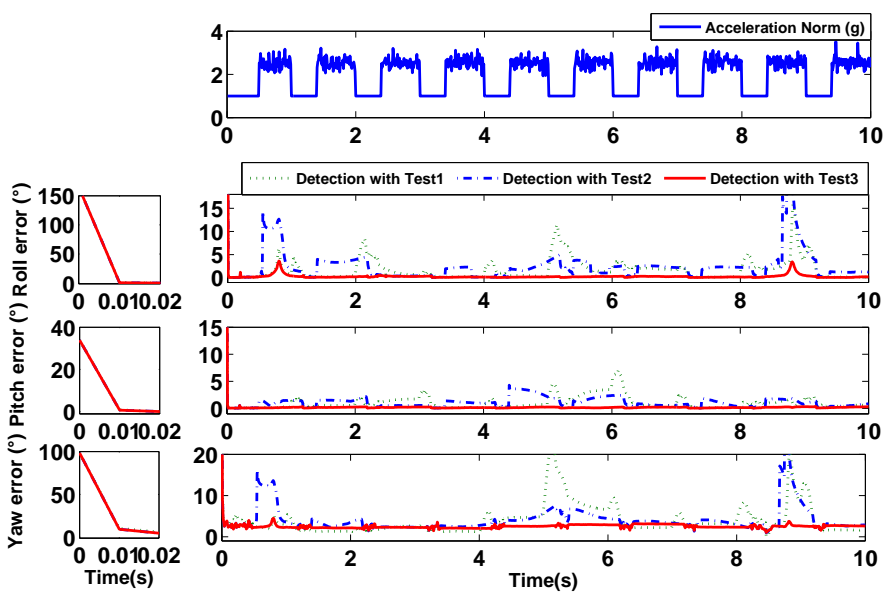

Fig. 6. Euler angles estimation errors in the case of low energy consumption mode

TABLE III

RMSE OF EULER ANGLES ESTIMATION (COMPARAISON OF THREE TESTS)

\begin{tabular}{cccc}
\hline \hline & Roll $\left(^{\circ}\right)$ & Pitch $\left(^{\circ}\right)$ & Yaw $\left(^{\circ}\right)$ \\
\hline Test 1 & 1.0558 & 1.4156 & 6.0135 \\
\hline Test 2 & 4.138 & 5.9742 & 3.4429 \\
\hline Test 3 & 0.3886 & 0.4689 & 3.5086 \\
\hline Full gyros & 0.5385 & 0.4513 & 1.5847 \\
\hline
\end{tabular}

\section{Filter performance in terms of energy consumption}

Now, let us evaluate the filter performance based on energy consumption criterion. For this purpose, we compute the current consumption of gyroscope during the same simulation period in each Test. For that, we define $A$ and $B$ as the current consumption when gyroscope is in active mode, sleep mode, and switching between the two previous modes, respectively. Then, the total energy consumption during the simulation period can be given by:

$$
E=n_{1} A+n_{2} B+n_{3} C,
$$

where $n_{1}, n_{2}, n_{3}$ stand for the number of samples when the gyroscope is in active mode, sleep mode and during switching between the two modes, respectively. The values of $A, B$ and $C$ can be found in the datasheet of the gyroscope. The threeaxis gyroscope L3GD20 is considered, as an example, and the values of $A$ and $B$ can be determined from [19] $(A=5.4 \mathrm{~mA}$ and $B=2.7 \mathrm{~mA}$ ). Sometimes, $C$ is not given in datasheets, however, we can easily consider that $C \ll A$ and $C \ll B$. Table IV shows the values of $n_{1}, n_{2}, n_{3}$, and $E$ in the three Tests. We conclude that the detector using acceleration norm (Test 1) gives the best results from an energy consumption point of view while the detector in Test 3 still have the highest performances in terms of detection and attitude estimation accuracy (see Table III). Moreover, the energy consumption is the same in both Test 2 and 3 but Test 3 gives better estimation performance since it avoids the non-detection cases (nondetection probability $=0$ due to the constraint $s_{1} \leq\left\|y_{a, k}\right\| \leq s_{2}$ ) as given in Table IV. 
TABLE IV

ENERGY CONSUMPTION IN THREE DETECTION CASES

\begin{tabular}{cccc}
\hline \hline & Test 1 & Test 2 & Test 3 \\
\hline$n_{1}$ & 590 & 760 & 761 \\
$n_{2}$ & 410 & 240 & 239 \\
$n_{3}$ & 25 & 19 & 19 \\
$E(m A)$ & $4293+25 C$ & $4752+19 C$ & $4754.7+19 C$ \\
Non-Detection Probability & & & \\
$($ PND) & 0.0034 & 0.0017 & 0 \\
\hline
\end{tabular}

\section{EXPERIMENTAL Results}

To assess the efficiency of q-AKF, several experiments were performed in the case of pedestrian walking for different scenarios. We used the foot-mounted MTi-IMU developed by Xsens Technologies [33] to collect measurements. The unit sampled sensor data for an orthogonally-oriented triaxial accelerometer, a triaxial gyroscope and a triaxial magnetometer at $100 \mathrm{~Hz}$. A set of experiments was carried out at the MOCA platform based in GIPSA-Lab at Univ. Grenoble Alpes to collect data for different users with different weights. The foot attitude is calculated by a Vicon motion capture system, containing 12 cameras T40s, through Tracker software. Vicon reconstructs the position and orientation of objects with passive markers that reflect light sent by the spotlight. The triad composed of markers is aligned with the one of MTi's sensors to synchronize later the q-AKF and the Vicon system. The MTi gyroscopes were calibrated before starting the experimental test. We kept the MTi at rest for a period of $10 \mathrm{~min}$ and we calculate the average of gyroscope measurements during this period, which corresponds to the gyroscope bias. Then, during the experiment, this gyroscope bias was subtracted from the gyroscope outputs at each sample time. Fig. 7 shows the overall experimental setup. All subjects were asked to walk on a path marked on the room, including rectangle shaped walking with a width of $2 \mathrm{~m}$ and a length of $3 \mathrm{~m}$ during 3 $\mathrm{min}$ as in Fig. 7. The collected data from the MTi are processed offline using the q-AKF implemented under MATLAB to estimate attitude in terms of quaternion representation and are compared with the attitude (in terms of quaternion also) calculated directly by the Vicon system which is considered as the reference. Two algorithms are considered: the QKF proposed by Choukroun et al. [14] and our approach based on the q-AKF. Similar results are obtained with all subjects for the same experiments. Then, we choose to represent one of these results in Fig. 8 where Euler angles estimation errors are plotted. We can see that the time evolution of estimation errors is globally more important when using the QKF filter as from the simulations test (previous Section). The dashed line curves, corresponding to the QKF, present significant peaks whenever external acceleration $a_{p, k}$ is detected. However, we note from the figure that the yaw estimation error is higher compared to roll and pitch estimation errors. This instantaneous error during the considered indoor scenario is caused by the presence of external magnetic distortions (ferromagnetic elements) between 16 and $28 \mathrm{sec}$ which impair only the yaw angle. When the magnetic distortions vanished between 28 and $40 \mathrm{sec}$, the error became less than $5^{\circ}$. In outdoor tests, these errors are

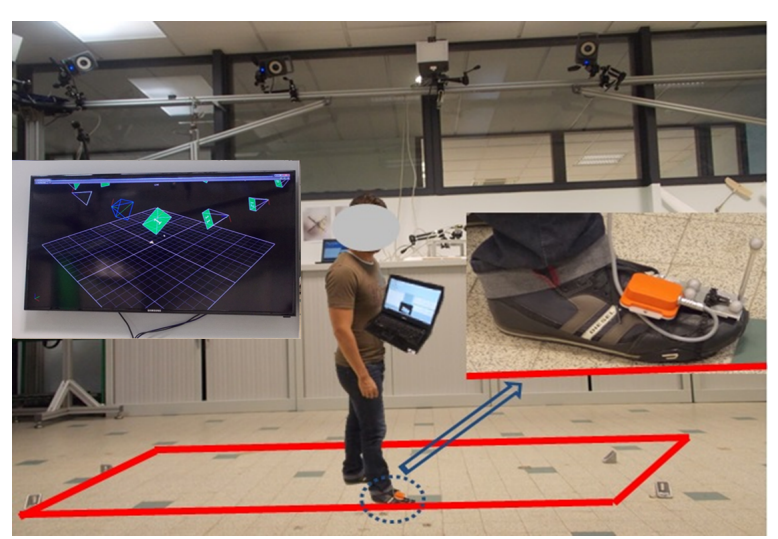

Fig. 7. Experimental setup
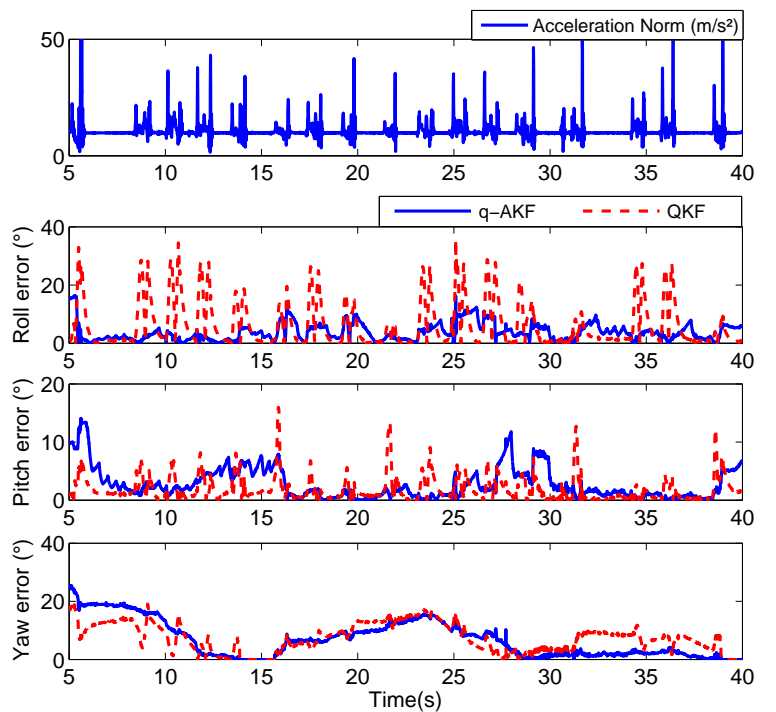

Fig. 8. Euler Angles estimation errors

not observed since the magnetic distortions are low.

To give better interpretation of the results, we plotted in Fig. 9 the Cumulative Distribution Function of estimation errors for Euler angles using both filters (q-AKF and QKF). We can
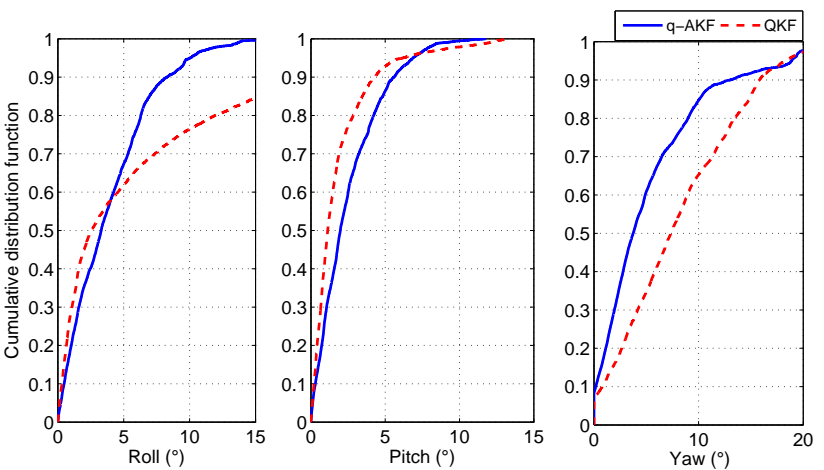

Fig. 9. Error Cumulative Distribution Function

note that estimation errors above $10^{\circ}$ for yaw angle are very rare events. $90 \%$ of time the estimation error is guaranteed 
to be less than $10^{\circ}$. We can also note that the q-AKF gives better performance than the QKF. For example, the probability to obtain an estimation error less than $5^{\circ}$ for the yaw angle is $35 \%$ with the QKF filter while it is about $60 \%$ with the q-AKF. We can remark also from the same figure that the main improvements are on roll and yaw angles estimation since external acceleration is important along the $\mathrm{x}$ (roll angle) and $y$ (yaw angle) axis of the MTi during the pedestrian walking scenario. This improvement on attitude estimation would enhance the precision on $3 \mathrm{D}$ position determination.

\section{CONCLUSION}

In this paper the design and some experimental results of a quaternion-based adaptive Kalman filter (q-AKF) for rigid body attitude estimation using inertial/magnetic sensors have been presented. The q-AKF was developped with two major goals: being able to produce accurate attitude estimates under external acceleration and minimal use of gyroscope measurements for energy consumption issue. The q-AKF doesn't need the setting of thresholds or the modelling of external acceleration. Based on the filter residual in the accelerometer, the external acceleration covariance matrix is estimated to adaptively tune the filter gain. The q-AKF was modified to find the best way to reduce the use of gyroscope measurements by turning-off and activating the sensor alternatively, while maintaining acceptable attitude estimation. The process noise covariance is adaptively tuned for optimal compensation of the error. A smart detection approach is proposed to decide whether the body is in dynamic or static case. Through numerical simulations and vicon-based experimental setup, under external acceleration and minimal gyroscope's use, the efficiency of the proposed q-AKF is illustrated. The presented approach doesn't address the problem of attitude estimation under magnetic disturbances which affect the yaw estimation accuracy. This issue will be the subject of future works.

\section{APPENDIX A}

Equation (10) defines the norm of accelerometer output under $H_{0}$. Let consider $x_{k}=\left\|y_{a, k}\right\|^{2}-g^{2}$. The variance of $x_{k}$ can be obtained as follows:

$$
\begin{aligned}
\operatorname{var}\left(x_{k}\right)= & E\left[x_{k}^{2}\right]-\left(E\left[x_{k}\right]\right)^{2} \\
= & E\left[\left\|\delta_{a, k}\right\|\left\|^{4}+4 g\right\| \delta_{a, k} \|^{2} u_{k}^{T} \delta_{a, k}\right. \\
& \left.+4 g^{2}\left(u_{k}^{T} \delta_{a, k}\right)^{2}\right]-9 \sigma^{4} \\
= & E\left[\left\|\delta_{a, k}\right\|^{4}\right]+4 g^{2} E\left[\left(u_{k}^{T} \delta_{a, k}\right)^{2}\right],
\end{aligned}
$$

with

$$
\begin{aligned}
E\left[\left\|\delta_{a, k}\right\|^{4}\right]= & E\left[\left(\delta_{a, k}^{x}\right)^{4}\right]+E\left[\left(\delta_{a, k}^{y}\right)^{4}\right]+E\left[\left(\delta_{a, k}^{z}\right)^{4}\right] \\
& +2 E\left[\left(\delta_{a, k}^{x}\right)^{2}\right] E\left[\left(\delta_{a, k}^{y}\right)^{2}\right]+2 E\left[\left(\delta_{a, k}^{x}\right)^{2}\right] E\left[\left(\delta_{a, k}^{z}\right)^{2}\right] \\
& +2 E\left[\left(\delta_{a, k}^{y}\right)^{2}\right] E\left[\left(\delta_{a, k}^{z}\right)^{2}\right]=15 \sigma^{4},
\end{aligned}
$$

$$
\begin{aligned}
E\left[\left(u_{k}^{T}\left(\delta_{a, k}\right)^{T}\right]\right. & =E\left[\left(u_{k}^{T}\left(\delta_{a, k}\right)^{T}\left(u_{k}^{T} \delta_{a, k}\right)\right]\right. \\
& =E\left[\operatorname{Tr}\left(\left(u_{k}^{T} \delta_{a, k}\right)\left(u_{k}^{T} \delta_{a, k}\right)^{T}\right)\right] \\
& =E\left[\operatorname{Tr}\left(u_{k}^{T} \delta_{a, k} \delta_{a, k}^{T} u_{k}\right)\right] \\
& =\operatorname{Tr}\left(E\left[u_{i} u_{k}^{T}\right] E\left[\delta_{a, k} \delta_{a, k}^{T}\right]\right. \\
& =\sigma^{2} E\left[\operatorname{Tr}\left(u_{i} u_{k}^{T}\right)\right]=\sigma^{2} .
\end{aligned}
$$

Mean value of $f_{k}$ :

$$
\begin{aligned}
E\left[f_{k}\right] & =E\left[\frac{1}{W} \sum_{i=k-W+1}^{k}\left(\left\|y_{a, i}\right\|^{2}-g^{2}-3 \sigma_{a}^{2}\right)\right] \\
& =\frac{1}{W} \sum_{i=k-W+1}^{k}\left(E\left[\left\|y_{a, i}\right\|^{2}-g^{2}-3 \sigma_{a}^{2}\right]\right) \\
& =\frac{1}{W} \sum_{i=k-W+1}^{k}\left(E\left[\left\|y_{a, i}\right\|^{2}\right]-\frac{1}{W} \sum_{i=k-W+1}^{k}\left(g^{2}+3 \sigma_{a}^{2}\right)=0 .\right.
\end{aligned}
$$

Variance of $f_{k}$ :

$$
\begin{aligned}
\sigma_{f}^{2} & =\frac{1}{W^{2}} \operatorname{var}\left(\sum_{i=k-W+1}^{k}\left(\left\|y_{a, i}\right\|^{2}-g^{2}-3 \sigma_{a}^{2}\right)\right. \\
& =\frac{1}{W^{2}} \sum_{i=k-W+1}^{k} \operatorname{var}\left(\left\|y_{a, i}\right\|^{2}-g^{2}-3 \sigma_{a}^{2}\right) \\
& =\frac{1}{W^{2}} \sum_{i=k-W+1}^{k} \operatorname{var}\left(\left\|y_{a, i}\right\|^{2}\right) \\
& =\frac{1}{W^{2}} \sum_{i=k-W+1}^{k}\left(6 \sigma_{a}^{4}+4 g^{2} \sigma_{a}^{2}\right)=\frac{1}{W}\left(6 \sigma_{a}^{4}+4 g^{2} \sigma_{a}^{2}\right) .
\end{aligned}
$$

\section{ACKNOWLEDGMENT}

The authors thank Jonathan Dumon who have made a great contribution to the tests achieved on the experimental platform funded by EquipeEx Robotex (ANR10-EQPX-44-01).

\section{REFERENCES}

[1] J.-K. Lee and E. J. Park, "Quasi real-time gait event detection using shank-attached gyroscopes." Med. Biol. Engineering and Computing, vol. 49, no. 6, pp. 707-712, 2011.

[2] D. Roetenberg, P. J. Slycke, and P. H. Veltink, "Ambulatory position and orientation tracking fusing magnetic and inertial sensing," IEEE Transactions on Biomedical Engineering, vol. 54, no. 5, pp. 883-890, 2007.

[3] X. Yun, E. Bachmann, H. Moore, and J. Calusdian, "Self-contained position tracking of human movement using small inertial/magnetic sensor modules," in IEEE International Conference on Robotics and Automation, April 2007, pp. 2526-2533.

[4] E. Bachmann, I. Duman, U. Y. Usta, R. Mcghee, X. Yun, and M. Zyda, "Orientation tracking for humans and robots using inertial sensors," in International Symposium on Computational Intelligence in Robotics and Automation, 1999, pp. 187-194.

[5] V. Renaudin and C. Combettes, "Magnetic, acceleration fields and gyroscope quaternion (MAGYQ)-based attitude estimation with smartphone sensors for indoor pedestrian navigation," Sensors, vol. 14, no. 12, pp. 22 864-22 890, 2014.

[6] E. Foxlin, "Inertial head-tracker sensor fusion by a complimentary separate-bias Kalman filter," in Proceedings of the Virtual Reality Annual International Symposium. Washington, DC, USA: IEEE Computer Society, 1996, pp. 185-194.

[7] G. Ligorio and A. Sabatini, "A novel kalman filter for human motion tracking with an inertial-based dynamic inclinometer," IEEE Transactions on Biomedical Engineering, vol. 62, no. 8, pp. 2033-2043, Aug 2015. 
[8] A. Vinod, A. Mahindrakar, S. Bandyopadhyay, and V. Muralidharan, "A deterministic attitude estimation using a single vector information and rate gyros," IEEE/ASME Transactions on Mechatronics, vol. 20, no. 5, pp. 2630-2636, Oct 2015.

[9] R. Zhu, D. Sun, Z. Zhou, and D. Wang, "A linear fusion algorithm for attitude determination using low cost MEMS-based sensors," Measurement, vol. 40, no. 3, pp. 322-328, 2007.

[10] H. Ren and P. Kazanzides, "Investigation of attitude tracking using an integrated inertial and magnetic navigation system for hand-held surgical instruments," IEEE/ASME Transactions on Mechatronics, vol. 17, no. 2, pp. 210-217, 2012.

[11] S. Bonnabel, "Left-invariant extended Kalman filter and attitude estimation," in 46th IEEE Conference on Decision and Control, 2007, pp. 1027-1032.

[12] P. Martin and E. Salaun, "Invariant observers for attitude and heading estimation from low-cost inertial and magnetic sensors," in 46th IEEE Conference on Decision and Control, 2007, pp. 1039-1045.

[13] H. Fourati, N. Manamanni, L. Afilal, and Y. Handrich, "Complementary Observer for Body Segments Motion Capturing by Inertial and Magnetic Sensors," IEEE/ASME Transactions on Mechatronics, vol. 19, no. 1, pp. 149-157, Feb. 2014.

[14] D. Choukroun, I. Y. Bar-Itzhack, and Y. Oshman, "Novel quaternion Kalman filter," IEEE Transactions on Aerospace and Electronic Systems, vol. 42, no. 1, pp. 174-190, 2006.

[15] J. W. Wei Li, "Effective adaptive Kalman filter for MEMSIMU/magnetometers integrated attitude and heading reference systems," Journal of Navigation, vol. 66, no. 01, pp. 99-113, January 2013.

[16] R. Munguia and A. Grau, "Attitude and heading system based on EKF total state configuration," in IEEE International Symposium on Industrial Electronics (ISIE), 2011, pp. 2147-2152.

[17] H. Rehbinder and X. Hu, "Drift-free attitude estimation for accelerated rigid bodies," Automatica, vol. 40, no. 4, pp. 653-659, Apr. 2004.

[18] J. K. Lee, E. Park, and S. Robinovitch, "Estimation of attitude and external acceleration using inertial sensor measurement during various dynamic conditions," IEEE Transactions on Instrumentation and Measurement, vol. 61, no. 8, pp. 2262-2273, 2012.

[19] STMicroelectronics, "Mems motion sensor:three-axis digital output gyroscope," STMicroelectronics, Tech. Rep., 2013.

[20] STMicroelectronics., "Mems digital output motion sensor ultra lowpower high performance three-axis nano accelerometer," STMicroelectronics, Tech. Rep., 2013.

[21] J. B. Kuipers, Quaternions and rotation sequences : a primer with applications to orbits, aerospace, and virtual reality. Princeton, NJ: Princeton Univ. Press, 1999.

[22] M. D. Shuster, "Survey of attitude representations," Journal of the Astronautical Sciences, pp. 439-517, oct 1993.

[23] S. Beeby, MEMS Mechanical Sensors. Artech House, 2004.

[24] D. Titterton and J. Weston, Strapdown Inertial Navigation Technology, 2nd ed. The American Institute of Aeronautics and Astronautics, 2004.

[25] NOAA. [Online]. Available: http://www.ngdc.noaa.gov

[26] A. Makni, H. Fourati, and A. Y. Kibangou, "Adaptive Kalman Filter for MEMS-IMU based Attitude Estimation under External Acceleration and Parsimonious use of Gyroscopes," in European Control Conference ECC, Strasbourg, France, Jun. 2014.

[27] B. Amidan, T. Ferryman, and S. Cooley, "Data outlier detection using the Chebyshev theorem," in IEEE Aerospace Conference, March 2005, pp. 3814-3819.

[28] D. Gebre-Egziabher, G. Elkaim, J. D. Powell, and B. Parkinson, "A gyro-free quaternion-based attitude determination system suitable for implementation using low cost sensors," in IEEE Position Location and Navigation Symposium, 2000, pp. 185-192.

[29] F. Zhou, Y. Wu, and N. Fan, "Improved accelerometer and magnetometer-based solution to solve quaternion," in 12th International Conference on Control, Automation and Systems (ICCAS), 2012. IEEE, 2012, pp. 2093-2096.

[30] J. Chou, "Quaternion kinematic and dynamic differential equations," IEEE Transactions on Robotics and Automation, vol. 8, no. 1, pp. 53-64, Feb 1992.

[31] J. F. Guerrero-Castellanos, H. Madrigal-Sastre, S. Durand, L. Torres, and G. A. Muoz-Hernndez, "A robust nonlinear observer for real-time attitude estimation using low-cost MEMS inertial sensors," Sensors, vol. 13, no. 11, pp. $15138-15158,2013$.

[32] W. F. Phillips, C. E. Hailey, and G. A. Gebert, "Review of attitude representations used for aircraft kinematics," J. Aircraft, vol. 38, no. 04, pp. 718-223, 2001.

[33] X. Technologies. [Online]. Available: http://www.xsens.com 